\title{
Technical Note: The Uses of I'mRT MatriXX in Electron Beams
}

\author{
Mutian Zhang, Sicong Li, Hua Deng, Sumin Zhou \\ Department of Radiation Oncology, University of Nebraska Medical Center, Omaha, USA \\ Email: mutianzhang@unmc.edu
}

Received Ocotber 20, 2012; revised November 22, 2012; accepted November 30, 2012

\begin{abstract}
Purpose: The objective of this study is to investigate the properties of I'mRT MatriXX device in electron beams, and to validate MatriXX in electron dosimetry and quality assurance (QA). Methods: The measurements were conducted using MatriXX in electron and photon beams from Siemens linacs. The MatriXX was placed horizontally on the linac tabletop. Solid Water layers were used for buildup. For all the measurements, the linac gantry angle was $0^{\circ}$, and the source-to-surface distance was $100 \mathrm{~cm}$ from the Solid Water surface. The electron cone factors, cutout factors, and beam profiles were measured and compared with thimble ionization chamber results. Results: The effective water equivalent depth of MatriXX measurement point is larger than $4 \mathrm{~mm}$. When measuring at the respective depths of maximum dose, MatriXX has different responses to different beam energies. The cone factors measured by MatriXX are nearly identical or close to those derived by ionization chambers. Beam profiles (flatness and symmetry) can be easily determined using MatriXX and are comparable to water tank results. The planar dose map of electron cutout blocks can be visually observed, and the cutout factors can be conveniently measured. Conclusions: The MatriXX needs separate dose calibration factors for electron and photon beams. MatriXX can be used to measure electron cutout factors and beam profiles, thus has the potentials in electron beam dosimetry and routine linac and patient-specific QA tests.
\end{abstract}

Keywords: Electron Beam; MatriXX; Dosimetry; Quality Assurance

\section{Introduction}

The I'mRT MatriXX (IBA Dosimetry GmbH, Germany) device consists of a two-dimensional (2D) array of ionization chambers. There are 1,020 vented parallel plate ion chambers on the array detector, arranged in $32 \times 32$ grid. The chamber center-to-center distance is $7.62 \mathrm{~mm}$, and the active area is $24.4 \times 24.4 \mathrm{~cm}^{2}$. MatriXX has been validated for 2D dose measurements [1], and is increasingly used in photon beam dosimetry and patient-specific quality assurance (QA) [2-4]. The application of MatriXX is also extended to QA checks for proton therapy [5].

In this work, we report our investigation on the feasibility of using MatriXX in electron beam dosimetry and routine linac QA or patient-specific treatment QA. This note is the expansion of an abstract submitted to the 2010 American Association of Physicists in Medicine annual meeting [6].

\section{Methods}

The measurements were conducted using electron and photon beams from Siemens ONCOR ${ }^{\mathrm{TM}}$ and PRIMUS ${ }^{\mathrm{TM}}$ linacs (Siemens Healthcare, Germany). The linacs could produce $6 \mathrm{MV}$ and $23 \mathrm{MV}$ photons, and electron beams with multiple energies between $5 \mathrm{MeV}$ and $21 \mathrm{MeV}$. The MatriXX was placed horizontally on the linac treatment couch, supported by 5 or $6 \mathrm{~cm}$ Solid Water ${ }^{\circledR}$ (Gammex Inc., USA) blocks (Figure 1). The MatriXX was positioned using the linac light field. On the MatriXX surface, $30 \mathrm{~cm} \times 30 \mathrm{~cm}$ Solid Water layers served as beam buildup with $1 \mathrm{~mm}$ thickness resolution. The linac gantry angle was $0^{\circ}$, and the source-to-surface distance was set at $100 \mathrm{~cm}$ from the Solid Water surface.

The MatriXX was previously calibrated for the photon beams. Before each use, the MatriXX was powered on for 30 minutes, and irradiated with at least $500 \mathrm{cGy}$ until stable readings were achieved. For each reading, 100 monitor units were delivered. The measurements of each data point were repeated three times. When taking the readings, the calibration factor of $6 \mathrm{MV}$ photon beam was used, so that the MatriXX responses to different beam energies could be compared. The chamber array was placed at the depth of maximum dose $\left(\mathrm{d}_{\max }\right)$ of the corresponding beam energy for the measurements of dose response, cone factor or cutout factor. The MatriXX measurements were compared with beam data acquired using calibrated PTW semiflex thimble chambers (PTW 


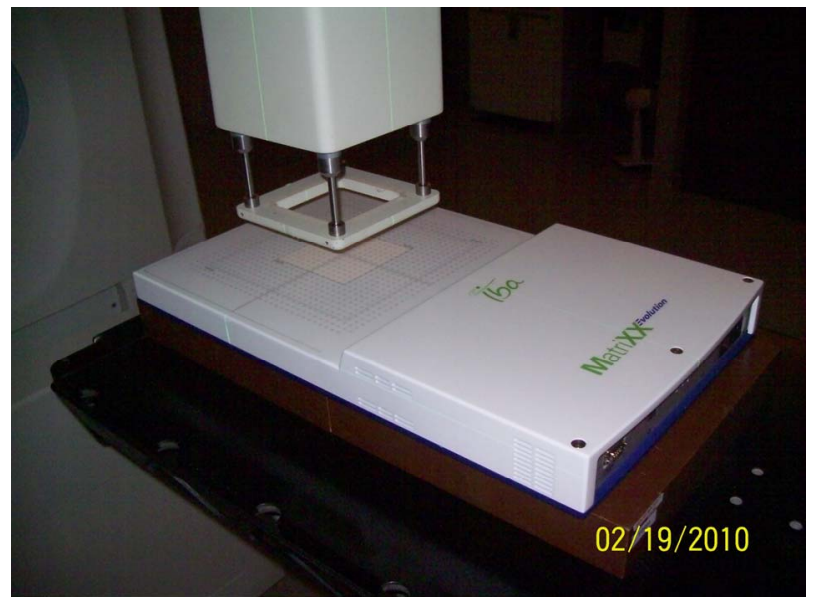

Figure 1. MatriXX setup on a linac treatment tabletop. A proper thickness of Solid Water would be placed on the surface during measurement.

Freiburg GmbH, Germany).

\section{Results and Discussion}

\subsection{Depth Ionization and Beam Profiles}

In order to determine the appropriate Solid Water thickness for the tasks carried out by the MatriXX, we measured the ionization curves for various electron and photon energies using MatriXX with Solid Water buildup at a depth resolution of $1 \mathrm{~mm}$, and normalized the data to the maximum value of each energy. The ionization curves acquired by ionization chambers were interpolated to find the depths of the percentage ionization in water corresponding to the thicknesses of Solid Water for the same percentage ionization of the same beam energies. It is easy to prove using the method of least-squares that, the average of the differences between the depths of the percentage ionization in water and the corresponding thick nesses of the Solid Water is equal to the depth of the MatriXX effective measurement point. Our measurements suggest that the effective measurement point of MatriXX is deeper than $4 \mathrm{~mm}$ water equivalent below the top surface. For instance, the best fit of MatriXX and ionization chamber data showed that the depth of effective measurement point of a specific MatriXX is $4.2 \mathrm{~mm}$ water equivalent for the $12 \mathrm{MeV}$ beam of an ONCOR linac (Figure 2). During the remaining measurements, the Solid Water thickness was determined with an approximate effective measurement depth of $4 \mathrm{~mm}$.

The MatriXX has the function to analyze beam profiles. Our results show that at the specified depths of photon $(10 \mathrm{~cm})$ and electron $\left(\mathrm{d}_{\max }\right)$ beams, the profiles of open fields measured with the MatriXX are nearly identical to those scanned with thimble chambers in a water tank. Therefore, the MatriXX provides a fast and convenient way to detect the changes in beams profiles. For

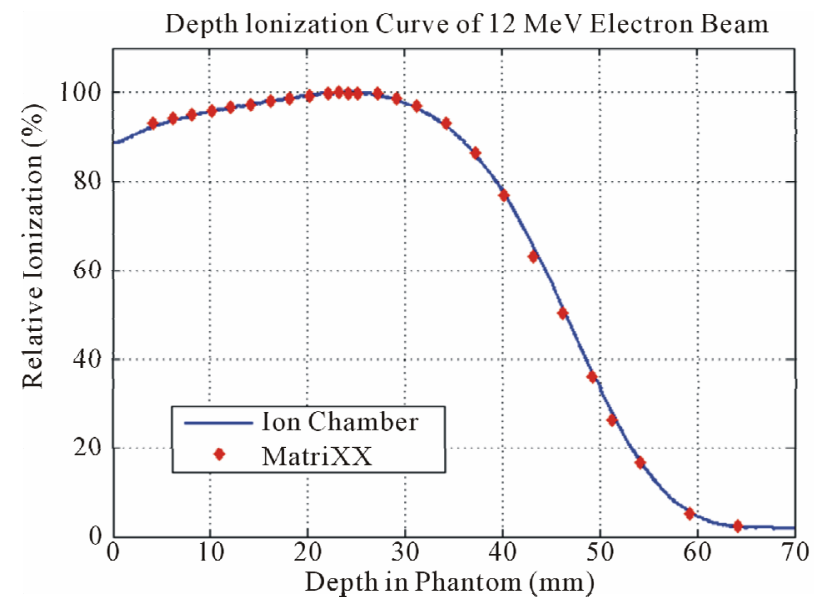

Figure 2. MatriXX and ionization chamber show close results in depth ionization measurement. The MatriXX data points are labeled with Solid Water thickness plus $4.2 \mathrm{~mm}$.

this reason, we are increasingly using MatriXX in the monthly linac QA to check the flatness and symmetry of electron beams as well as that of photons. We must stress that MatriXX is useful in checking the beam profile consistency, but it cannot replace scanning water tank in the measurement of beam profiles.

\subsection{MatriXX Response to Electron Beams}

We observed that, the reproducibility of MatriXX reading was comparable to that of a thimble chamber. The ionization of electron energies was measured with MatriXX at $d_{\max }$ using $10 \mathrm{~cm} \times 10 \mathrm{~cm}$ cones and compared with photon beams of the same field size. The readings of MatriXX central chambers were corrected with the thimble chamber measurements of the linac output factors on the same day, and normalized to the $6 \mathrm{MV}$ reading for the same dose at $d_{\max }$. Our data showed that the ionization chambers in MatriXX have different dose response to electron beams from that to photon beams. Table 1 shows the relative response of MatriXX to photon and electron beams of a PRIMUS linac. Our results suggest that the MatriXX needs a calibration factor for the electron energy in question, especially when patientspecific treatment plan QA is conducted [7].

\subsection{Electron Beam Dosimetry}

The output factors of electron cones were measured at the depth of the maximum dose. These factors were normalized to the $10-\mathrm{cm}$ cone output of the corresponding energies, and most matched the water tank ionization chamber measurements very well (Table 2). For reasons yet unknown, some data showed larger discrepancies, up to $0.9 \%$.

The cutout factors of square inserts in $10-\mathrm{cm}$ cone were measured with MatriXX. For the 3-cm and 2-cm 
Table 1. The relative response of a MatriXX to clinical photon and electron beams of a PRIMUS linac. The readings were normalized to $6 \mathrm{MV}$ photon energy using the actual linac output of each modality.

\begin{tabular}{cccccccc}
\hline Beam Energy & $6 \mathrm{X}$ & $23 \mathrm{X}$ & $6 \mathrm{MeV}$ & $9 \mathrm{MeV}$ & $12 \mathrm{MeV}$ & $15 \mathrm{MeV}$ & $18 \mathrm{MeV}$ \\
Normalized Reading & 1.000 & 0.993 & 1.070 & 1.069 & 1.068 & 1.077 & 1.101 \\
\hline
\end{tabular}

Table 2. The electron cone factors measured with MatriXX (MX) and ionization chamber (IC) on a PRIMUS linac. The 5-cm cone is circular while the rest cones are square (\%diff. = percentage difference).

\begin{tabular}{ccccccccccccccccc}
\hline Energy & \multicolumn{3}{c}{$6 \mathrm{MeV}$} & \multicolumn{3}{c}{$9 \mathrm{MeV}$} & \multicolumn{3}{c}{$12 \mathrm{MeV}$} & \multicolumn{3}{c}{$15 \mathrm{MeV}$} & \multicolumn{3}{c}{$18 \mathrm{MeV}$} \\
\hline Cone size & MX & IC & \%diff. & MX & IC & \%diff. & MX & IC & \%diff. & MX & IC & \%diff. & MX & IC & \%diff. \\
\hline $5 \mathrm{~cm}$ (cir) & 0.787 & 0.789 & $-0.3 \%$ & 0.879 & 0.886 & $-0.8 \%$ & 0.920 & 0.920 & $0.0 \%$ & 0.942 & 0.942 & $0.0 \%$ & 0.968 & 0.968 & $0.0 \%$ \\
$10 \mathrm{~cm}$ & 1.000 & 1.000 & NA & 1.000 & 1.000 & NA & 1.000 & 1.000 & NA & 1.000 & 1.000 & NA & 1.000 & 1.000 & NA \\
$15 \mathrm{~cm}$ & 1.018 & 1.016 & $0.2 \%$ & 0.993 & 0.992 & $0.1 \%$ & 0.995 & 0.993 & $0.2 \%$ & 1.001 & 1.001 & $0.0 \%$ & 1.001 & 1.001 & $0.0 \%$ \\
$20 \mathrm{~cm}$ & 1.029 & 1.026 & $0.3 \%$ & 0.973 & 0.973 & $0.0 \%$ & 0.964 & 0.961 & $0.3 \%$ & 0.969 & 0.967 & $0.2 \%$ & 0.962 & 0.966 & $-0.4 \%$ \\
$25 \mathrm{~cm}$ & 1.018 & 1.009 & $0.9 \%$ & 0.965 & 0.964 & $0.1 \%$ & 0.960 & 0.956 & $0.4 \%$ & 0.973 & 0.968 & $0.5 \%$ & 0.963 & 0.967 & $-0.4 \%$ \\
\hline
\end{tabular}

cutout, a MatriXX central chamber was placed at the center of the field for accurate readings. These inserts were made for electron beam commissioning and their cutout factors had been measured with a thimble chamber in water phantom. Table 3 compares the cutout factors for the ONCOR $12 \mathrm{MeV}$ beam from MatriXX and thimble chamber. The data suggest that the MatriXX may be a useful tool for the measurement of electron cutout factor. In most cases, the MatriXX can provide clinically acceptable cutout factors, and we routinely use the MatriXX to measure electron cutout factors. However, the MatriXX seems to underestimate the cutout factors of very small inserts (nearly 1\%). This tendency could be caused by the MatriXX air cavities, which make the differences in lateral scattering and attenuation of electrons. The accuracy of MatriXX for very small electron cutouts may need further investigation.

With Solid Water buildup of appropriate thickness, the MatriXX can provide a 2D dose map at the desired depth. This ability provides a convenient way to visualize electron dose distribution within a phantom. The 2D dose profile can help the clinician to judge whether a custom cutout block can provide enough coverage of the lesion to be treated (Figure 3). This is a rather useful feature when the electron treatment plan is based on clinical setup and without a 3D image.

\section{Conclusion}

The MatriXX responds differently to electron beams and photon beams, thus separate dose calibration factors should be established for electron dosimetry. It has been shown that MatriXX can be used to obtain electron cutout factors. The ability of MatriXX to display planar

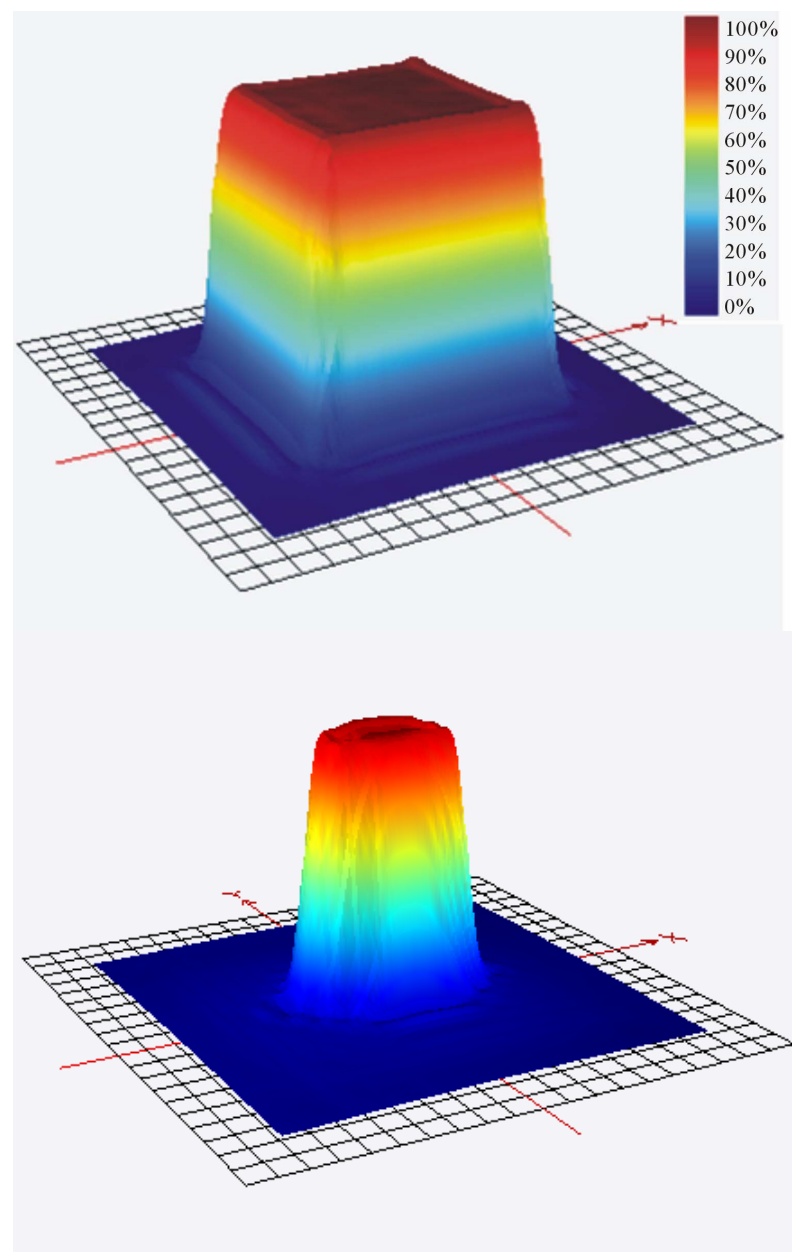

Figure 3. The MatriXX 2D electron beam profiles of $10 \mathrm{~cm}$ open field (top) and a custom cutout (bottom) at $d_{\max }$ with Solid Water buildup. The beam energy is $18 \mathrm{MeV}$, and the profiles are normalized to the same nominal dose. 
Table 3. Electron cutout factors of an ONCOR $12 \mathrm{MeV}$ beam measured with MatriXX and thimble chamber (\%diff. $=$ percentage difference).

\begin{tabular}{cccc}
\hline Cutout $\left(\mathrm{cm}^{2}\right)$ & MatriXX & Thimble Chamber & $\%$ diff. \\
\hline $10 \times 10$ & 1.000 & 1.000 & NA \\
$8 \times 8$ & 1.002 & 1.006 & $-0.4 \%$ \\
$6 \times 6$ & 0.992 & 0.992 & $0.0 \%$ \\
$4 \times 4$ & 0.938 & 0.943 & $-0.5 \%$ \\
$3 \times 3$ & 0.903 & 0.910 & $-0.8 \%$ \\
$2 \times 2$ & 0.870 & 0.878 & $-0.9 \%$ \\
\hline
\end{tabular}

dose map provides a useful tool in beam profile measurement. MatriXX has the potentials in electron beam dosimetry and routine QA checks.

\section{REFERENCES}

[1] J. Herzen, M. Todorovic, F. Cremers, V. Platz, D. Albers, A. Bartels and R. Schmidt, "Dosimetric Evaluation of a 2D Pixel Ionization Chamber for Implementation in Clinical Routine," Physics in Medicine and Biology, Vol. 52, No. 4, 2007, pp. 1197-1208. doi:10.1088/0031-9155/52/4/023
[2] J. G. Li, G. Yan and C. Liu, "Comparison of Two Commercial Detector Arrays for IMRT Quality Assurance," Journal of Applied Clinical Medical Physics, Vol. 10, No. 2, 2009, pp. 63-74. doi:10.1120/jacmp.v10i2.2942

[3] E. Schreibmann, A. Dhabaan, E. Elder and T. Fox, "Patient-Specific Quality Assurance Method for VMAT Treatment Delivery," Medical Physics, Vol. 36, No. 10, 2009, pp. 4530-4535. doi:10.1118/1.3213085

[4] J. O'Daniel, S. Das, Q. J. Wu and F. F. Yin, "Volumetric-Modulated Arc Therapy: Effective and Efficient Endto-End Patient-Specific Quality Assurance," International Journal of Radiation Oncology, Biology, Physics, Vol. 82, No. 5, 2012, pp. 1567-1574.

doi:10.1016/j.ijrobp.2011.01.018

[5] B. Arjomandy, N. Sahoo, X. Ding and M. Gillin, "Use of a Two-Dimensional Ionization Chamber Array for Proton Therapy Beam Quality Assurance," Medical Physics, Vol. 35, No. 9, 2008, pp. 3889-3894. doi:10.1118/1.2963990

[6] M. Zhang, S. Li, H. Deng and S. Zhou, "The Applications of MatriXX to Electron Beam Dosimetry," Medical Physics, Vol. 37, No. 6, 2007, p. 3268. doi:10.1118/1.3468756

[7] F. Rosca, "A Hybrid Electron and Photon IMRT Planning Technique That Lowers Normal Tissue Integral Patient Dose Using Standard Hardware," Medical Physics, Vol. 39, No. 6, 2012, pp. 2964-2971. doi:10.1118/1.4709606 